\title{
Nouveaux à la rédaction du BMS
}

\section{Bruno Kesseli}

Dr méd. et lic. phil., rédacteur en chef

Le cercle de la rédaction du BMS s'agrandit de quatre nouveaux membres: Urs Brügger, Ursina Pally, Milo Puhan et Charlotte Schweizer. La rédaction est convaincue que l'expertise des nouveaux membres de la rédaction sera bénéfique pour la qualité de la revue et profitera donc aux lectrices et lecteurs du BMS. Nous nous réjouissons à la perspective de cette collaboration.

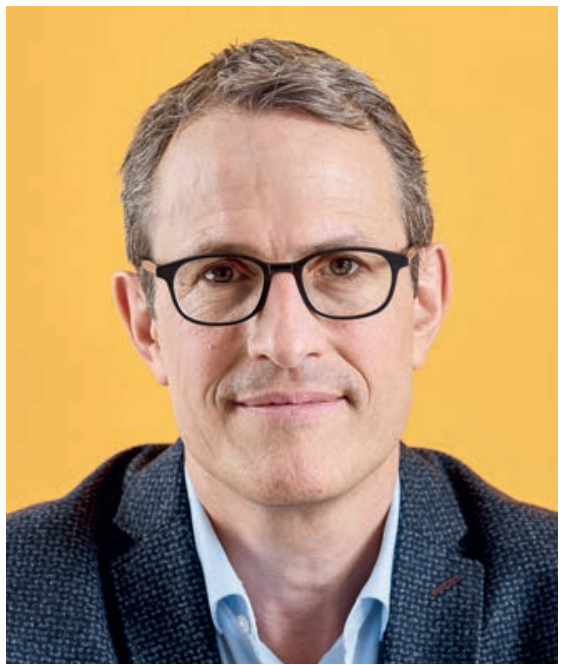

Urs Brügger a étudié l'économie et la sociologie à l’Université de Saint-Gall. Il est titulaire d'un doctorat en économie, d'un Master in Health Technology Assessment and Management et de formations complémentaires en économie de la santé, didactique, leadership et coaching. De 2003 à 2017, il a dirigé l'Institut d'économie de la santé de Winterthour à l'Université des sciences appliquées de Zurich (ZHAW). Depuis le début de l'année 2018, il est directeur du département de santé de la Haute école spécialisée bernoise (BFH). Depuis 2013 il est membre du comité de direction et questeur de l'Académie Suisse des Sciences Médicales ASSM. Au sein de la rédaction du BMS, il succède à Anna Sax, qui s'était retirée fin 2017 pour prendre en 2018 la direction de l'office de la santé du canton de Schaffhouse.

\section{Ursina Pally Hofmann}

Ursina Pally Hofmann dirige depuis juin de cette année le service juridique de la FMH. Après une formation initiale de sage-femme et dix ans d'expérience professionnelle pratique, elle a étudié le droit à l'Université de Zurich, obtenu son doctorat en droit, été admise au barreau et a complété sa formation par un "CAS Health Systems» des universités de Zurich, Bâle et Berne. Elle a travaillé pendant une dizaine d'années en tant qu'avocate dans différents domaines, notamment à la FMH en tant que directrice adjointe du service juridique, à des postes de direction dans une grande compagnie d'assurances et en tant que professeur de haute école et juge commerciale. Elle continue d'exercer ces deux dernières activités en parallèle de sa fonction principale. Au BMS, elle succède à Hanspeter Kuhn en tant que rédactrice spécialisée dans le droit.

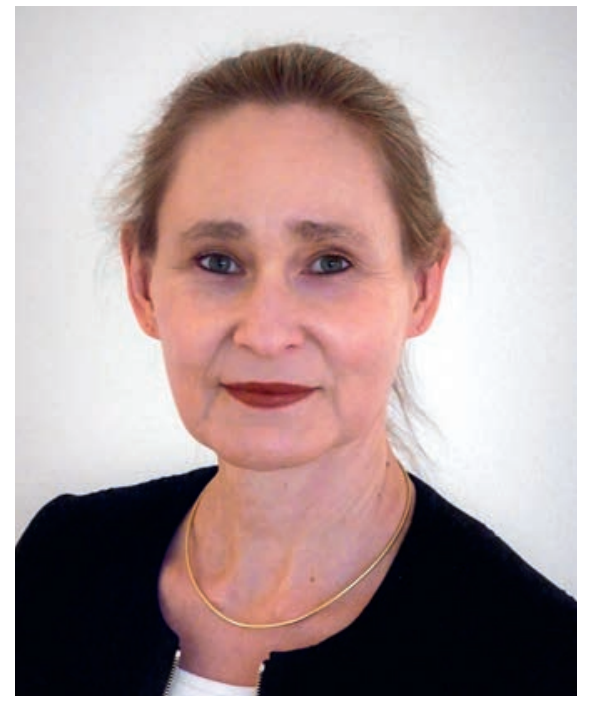


Milo Puhan

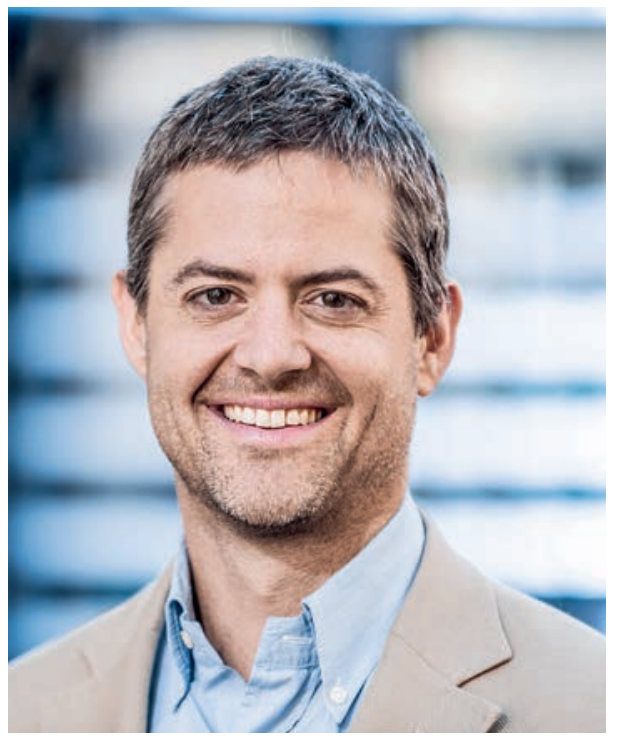

Milo Puhan a étudié la médecine à l'Université de Zurich, avant de travailler comme médecin-assistant, puis comme Research Fellow au Centre Horten de l'UZH. Il a obtenu son doctorat en épidémiologie à l'Université d'Amsterdam. De 2008 à 2012, il a été Associate Professor avec Tenure Track au Department of Epidemiology de la Johns Hopkins Bloomberg School of Public Health. Depuis 2013, il est professeur ordinaire d'épidémiologie et de santé publique à l'Université de Zurich, où il assume également les fonctions de directeur de l'Institut d'épidémiologie, de biostatistique et de prévention. Dans la recherche, Milo Puhan s'intéresse principalement à la prévention et à la gestion des maladies chroniques, ainsi qu'au développement d'outils qui favorisent les soins de santé basés sur les préférences. A la rédaction du BMS, Milo Puhan est chargé du domaine «Santé publique, épidémiologie et biostatistiques» en tant que rédacteur spécialisé.

\section{Charlotte Schweizer}

Charlotte Schweizer dirige la division Communication de la FMH depuis février de cette année. Après ses études des langues et littératures anglaise et française à l'Université de Bâle, elle a exercé différentes fonctions au sein du groupe Coop, notamment celle de cheffe de projet dans le service de presse. Elle a ensuite travaillé comme porte-parole au Comité suisse de l'UNICEF, le Fonds des Nations Unies pour l'enfance. En outre, elle a suivi en cours d'emploi des études de sciences de la communication et des médias à la Haute école d'économie de Zurich (HWZ) et à l'Ecole suisse de journalisme de Lucerne (MAZ). Charlotte Schweizer est membre de l'équipe de rédaction externe du BMS et succède donc à Jacqueline Wettstein.

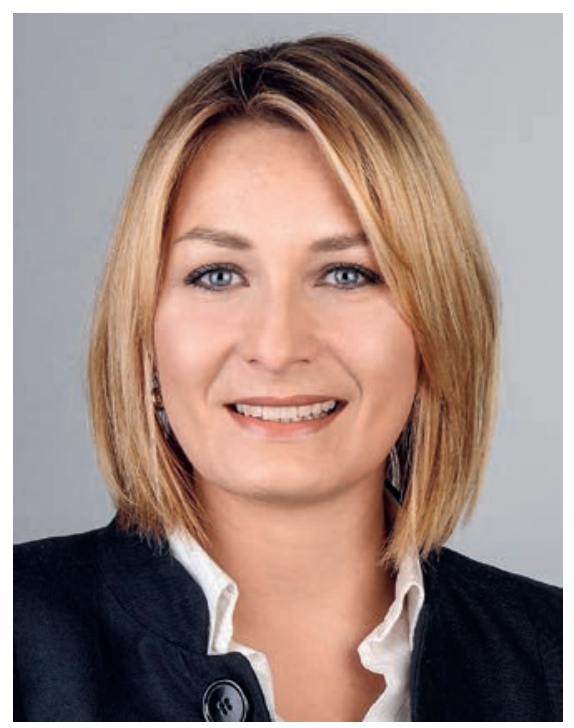

